This is a self-archived version of an original article. This version may differ from the original in pagination and typographic details.

Author(s): Kövener, T.; Küchler, D.; Toivanen, V. A.

Title: Lead evaporation instabilities and failure mechanisms of the micro oven at the GTS-LHC ECR ion source at CERN

Year: 2020

Version: Published version

Copyright: ㄷ 2020 Authors.

Rights: In Copyright

Rights url: http://rightsstatements.org/page//nC/1.0/?language=en

Please cite the original version:

Kövener, T., Küchler, D., \& Toivanen, V. A. (2020). Lead evaporation instabilities and failure mechanisms of the micro oven at the GTS-LHC ECR ion source at CERN. Review of Scientific Instruments, 91(1), Article 013320. https://doi.org/10.1063/1.5126084 


\section{Lead evaporation instabilities and failure mechanisms of the micro oven at the GTS- LHC ECR ion source at CERN}

Cite as: Rev. Sci. Instrum. 91, 013320 (2020); https://doi.org/10.1063/1.5126084

Submitted: 29 August 2019 . Accepted: 02 January 2020 . Published Online: 17 January 2020

T. Kövener (D), D. Küchler (D), and V. A. Toivanen (D)

\section{COLLECTIONS}

Paper published as part of the special topic on Proceedings of the 18th International Conference on Ion Sources Note: Contributed paper, published as part of the Proceedings of the 18th International Conference on Ion Sources, Lanzhou, China, September 2019.
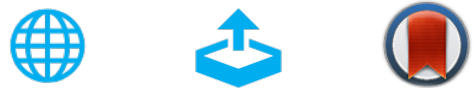

\section{ARTICLES YOU MAY BE INTERESTED IN}

ECRIS plasma spectroscopy with a high resolution spectrometer

Review of Scientific Instruments 91, 013318 (2020); https://doi.org/10.1063/1.5128854

${ }^{96} \mathrm{Zr}$ beam production for isobar experiment in relativistic heavy ion collider

Review of Scientific Instruments 91, 013319 (2020); https://doi.org/10.1063/1.5128618

A novel high power vacuum diode with a focusing electrode for effective operation in a low guiding magnetic field

Review of Scientific Instruments 91, 014706 (2020); https://doi.org/10.1063/1.5126691

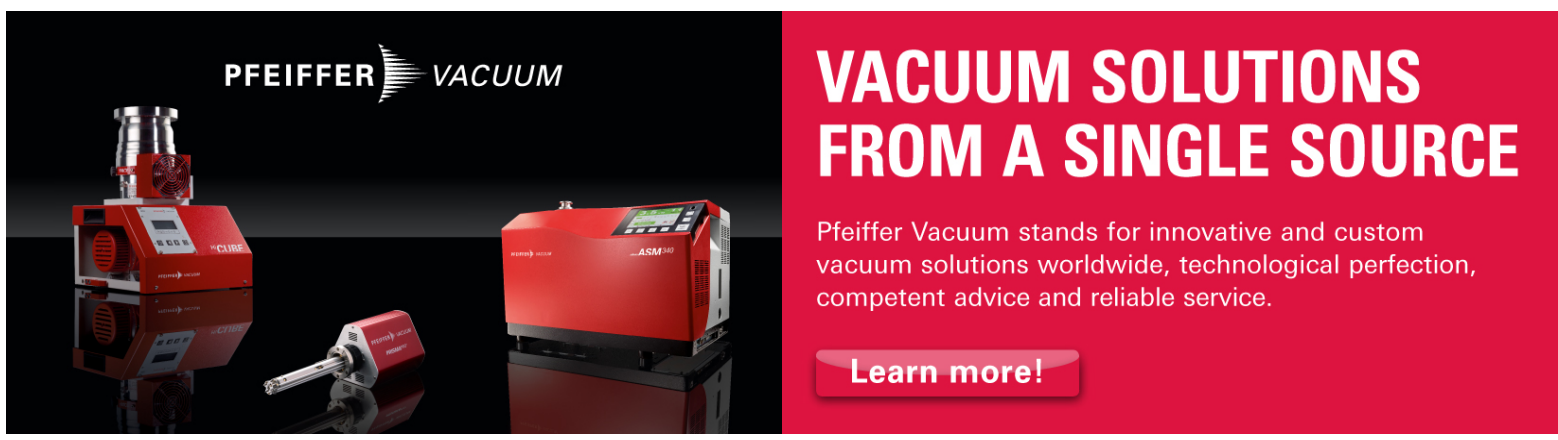




\title{
Lead evaporation instabilities and failure mechanisms of the micro oven at the GTS-LHC ECR ion source at CERN
}

Cite as: Rev. Sci. Instrum. 91, 013320 (2020); doi: 10.1063/1.5126084

Submitted: 29 August 2019 • Accepted: 2 January 2020 •

Published Online: 17 January 2020

T. Kövener, $^{1,2, a)}$ (D) D. Küchler, ${ }^{1}$ (D) and V. A. Toivanen ${ }^{3}$

\author{
AFFILIATIONS \\ ${ }^{1}$ European Organization for Nuclear Research (CERN), CH-1211 Geneva 23, Switzerland \\ ${ }^{2}$ University of Hamburg, 20146 Hamburg, Germany \\ ${ }^{3}$ University of Jyväskylä, Department of Physics (JYFL), 40500 Jyväskylä, Finland
}

Note: Contributed paper, published as part of the Proceedings of the 18th International Conference on Ion Sources, Lanzhou, China, September 2019.

a) Author to whom correspondence should be addressed: toke.koevener@cern.ch

\begin{abstract}
The GTS-LHC ECR ion source (named after the Grenoble Test Source and the Large Hadron Collider) at CERN provides heavy ion beams for the chain of accelerators from Linac3 up to the LHC for high energy collision experiments and to the Super Proton Synchrotron for fixed target experiments. During the standard operation, the oven technique is used to evaporate lead into the source plasma to produce multiple charged lead ion beams. Intensity and stability are key parameters for the beam, and the operational experience is that some of the source instabilities can be linked to the oven performance. Over long operation periods of several weeks, the evaporation is not stable which makes the tuning of the oven unpredictable and nonreproducible. A dedicated test stand is used to study the oven performance and possible improvements independently of the source operation. It was observed that the measured evaporation rate of the oven can vary spontaneously in a wide range even when stable operating conditions are applied to the oven controls. Data collected at the test stand hint that these fluctuations are caused by temperature instabilities of the oven itself. Several ways to improve the oven stability were tested, including insulation changes and modifications of the oven crucible. Some of the most promising results regarding the stability of the evaporation will be presented in this paper.
\end{abstract}

Published under license by AIP Publishing. https://doi.org/10.1063/1.5126084

\section{INTRODUCTION}

The CERN accelerator chain is relying on a constant production of heavy ions, usually lead ions that are being produced by the GTS-LHC ion source. ${ }^{1}$ The lead vapor ionized in the source plasma is coming from a micro-oven heating a metallic lead sample, which needs to be replaced approximately every second week. The temporal behavior of the oven performance during operation is, up to now, not fully understood. Determining which factors influence the oven stability and endurance can help to optimize the operation of the GTS-LHC ion source, especially with respect to long and stable times of uninterrupted lead ion production. For this purpose, a dedicated test stand allows us to measure the evaporation rate in realistic operation conditions and to test modifications to the oven setup.
The oven test stand (OTS), first presented in Ref. 2, is a simplified vacuum enclosure that features several feedthroughs, one for the oven and others for sensors and gas injection. It allows temperature measurements with thermocouples and evaporation rate measurements with an INFICON deposition detector. ${ }^{3}$ The deposition rate of the sensor is calibrated with the mass difference of the crucible before and after the measurement to calculate the evaporation rate. The oven is operated with a feedback loop that levels the heating power at a set point by controlling the power supply.

\section{EVAPORATION RATE INSTABILITIES}

Several previously reported measurements at the oven test stand have exhibited unpredicted changes in the evaporation rate. ${ }^{4}$ 


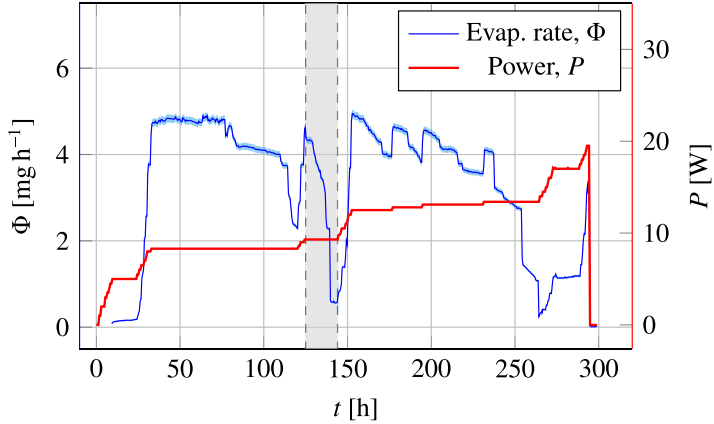

To rule out a number of possible influences, the oven setup was renewed by installing a new filament, inserting a previously unused crucible, and also refitting the heat reflection foil. The lead sample was produced from chemically clean lead (no chemical impurities but not isotopically purified). The oven was operated in a residual gas atmosphere of $1 \times 10^{-8}$ mbar. During the measurement, the power was ramped up to a comparable value at which the oven is operated at the GTS-LHC ion source at the beginning of a run $(9 \mathrm{~W})$. The oven power was then manually increased when the deposition rate dropped notably, with the goal to uphold a constant evaporation rate.

The left plot of Fig. 1 shows the resulting evaporation rate together with the applied heating power.

At a power of $9 \mathrm{~W}$, the evaporation rate increased to a value of around $5 \mathrm{mg} \mathrm{h}^{-1}$ and stayed stable for more than $60 \mathrm{~h}$ at a constant power value. Then, it dropped suddenly, which led to the decision to increase the oven power. Several drops followed, and by again adjusting the oven power, the evaporation rate could be prevented from dropping below $3 \mathrm{mg} \mathrm{h}^{-1}$ for approximately $80 \mathrm{~h}$. After a further drop was observed, it was decided to stop the measurement.

The time window of one distinctive drop is marked in gray in the left plot of Fig. 1. It was noted that during this and several other drops of the evaporation rate also, the heating filament resistance changed. The two plots on the right of Fig. 1 show parameters during the marked time window in the left plot. During this time, the heating power was not changed and remained at $9.3 \mathrm{~W}$. The top one again shows the evaporation rate. On the bottom, one the filament resistance in the same time frame is given. It shows a clear correlation. This correlation between the drop of the evaporation rate and the decrease in the filament resistance, while the heating power remains stable, has been observed in several other measurements. During this run, no thermocouples were attached to the oven to exclude them as a possible influence on the stability.

\section{INSULATION MODIFICATIONS}

Different changes to the oven setup have been tested with emphasis on the stability of the evaporation rate. Modifications of the thermal insulation showed a notable influence on the evaporation rate stability. The results of two modifications are presented here.

In one configuration, the oven was equipped with additional heat reflective insulation layers at the front as it was shown by Lang et al. ${ }^{5}$ that this can have a positive effect on the temperature homogeneity within the oven. These layers consisted of ring shaped tantalum foil pieces with an inner opening diameter of $3.6 \mathrm{~mm}$ at the tip of the crucible.

The results are shown in the left plot of Fig. 2. After ramping up the power, the evaporation rate could be held around an average of around $3 \mathrm{mg} \mathrm{h}^{-1}$ over the duration of the complete run, which lasted $350 \mathrm{~h}$. An initial overshoot of the evaporation was compensated by reducing the oven power. Initially, the evaporation rate stayed relatively stable for around $24 \mathrm{~h}$ and then started to show fluctuations.

Several times the evaporation rate dropped around $0.5 \mathrm{mg} \mathrm{h}^{-1}$ and then recovered to its previous value again. After around $90 \mathrm{~h}$ of evaporation, further drops occurred that would not recover and needed adjustment of the oven power.

The second configuration tested the evaporation rate stability with an oven setup where the thermal insulation was reduced. Here, a reflective tantalum foil that is present in the usual setup was removed. In this measurement, presented in the right plot of Fig. 2, the oven power had to be ramped up to $29.3 \mathrm{~W}$
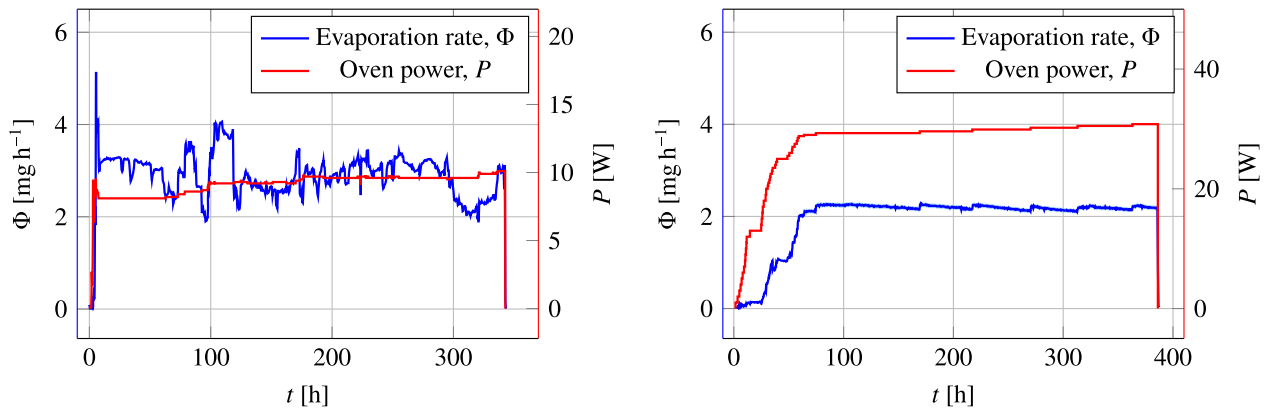

FIG. 2. Left plot: Evaporation rate and applied oven power with additional thermal insulation in the front of the oven. Right plot: The same values for a measurement, where the insulation was reduced by removing the heat reflective foil from the setup. 
to achieve a sufficient evaporation rate, which is much higher than what is usually necessary (between $8 \mathrm{~W}$ and $20 \mathrm{~W}$ ). Throughout the measurement, the power was then increased several times reaching $30.8 \mathrm{~W}$ after $360 \mathrm{~h}$. The measurement was stopped after $380 \mathrm{~h}$. No fluctuations of the evaporation rate were observed; after each increase in the power, it increased and then slowly decreased over time.

\section{DISCUSSION OF THE INSTABILITIES AND INSULATION MODIFICATIONS}

The linear correlation between the evaporation rate changes and the resistance changes shown in Fig. 1 is a hint that a process is influencing both the evaporation and the filament. A possible connection is the temperature. If the inner temperature of the oven changes for some reason, both the evaporation rate and the resistance will change.

The measurements with modified thermal insulation strengthen this interpretation. The measurement with increased insulation showed a fluctuating evaporation rate, while the reduced insulation led to a more stable behavior. If the fluctuations would solely be caused by a spatial inhomogeneous temperature distribution within the crucible, one could expect that the oven is more stable when the insulation is increased. As the opposite was the case, it can be deduced that the general thermal stability of the oven over time plays a greater role. A possible reason for this thermal instability is changes of the insulation properties during operation, possibly initiated by thermal stress on the heat reflective tantalum foil. The foil is only loosely inserted and could change its shape within the oven.

It cannot be ruled out that also other processes are present that influence both the filament resistance and the heating effect such as changes of the electrical properties of the filament, e.g., by evaporation. However, degradation of the filament should not directly affect the oven heating as the feedback levels the heating power and regulates the current accordingly. Especially for the test with the additional tantalum layers, it is also possible that a part of the observed instabilities has its origin in lead condensate accumulations at the orifice of the oven. However, these were not present during the measurement presented in Fig. 1.

\section{OPERATION IN AN OXIDIZING ATMOSPHERE}

The GTS-LHC ion source uses oxygen as a buffer gas during the lead ion production. When oxygen is present, lead condensate at the outer oven cover can be oxidized and form a blockage that is able to reduce or stop the flow of lead vapor from the oven. This process could also be observed and studied at the test stand, as presented in Ref. 4 . When oxygen gas with a pressure of $1 \times 10^{-5} \mathrm{mbar}$ is present in the OTS, the oven reproducibly formed a lead oxide cone that would block the oven completely within two weeks of operation.

It was tested if modifications to the oven setup can help to prevent the formation of such a blockage. As the starting point of the lead oxide formation is the accumulation of lead condensate on the oven cover, the focus was on the prevention of the condensate formation. Here, two approaches are possible: to heat up the outer oven cover until the local vapor pressure is high enough so that incident lead vapor does not condensate or to minimize the amount of vapor that is deposited on the oven cover.
To operate the oven without the reflective tantalum foil, as presented in the section titled "Discussion of the instabilities and insulation modifications," makes it necessary to use higher heating powers than in the usual setup. The higher heating power for the same crucible temperature leads to a hotter outer oven cover (around $450{ }^{\circ} \mathrm{C}$ instead of values around $300^{\circ} \mathrm{C}$ ). It was observed that without the foil the oven could be operated within $1 \times 10^{-5}$ mbar of oxygen, without any kind of oxide formation at the oven tip until the crucible was completely empty. Again the evaporation rate showed no fluctuations. A comparison of the appearance of the oven tip of different runs after operating the oven within oxygen can be seen in Fig. 3.

Another modification was developed to prevent the condensate formation even when the oven cover is colder, like in the usual oven setup. Here, the crucible design is changed by extending its tip outside of the oven cover with a cylindrical beak. This way the lead vapor is only incident on oven surfaces that are hot enough to prevent condensation. Figure 4 sketches the principle of adding the beak to the crucible to prevent the lead deposition on the oven cover.

The design was tested at the oven test stand by operating the oven within an oxygen atmosphere of $1 \times 10^{-5} \mathrm{mbar}$. Here, the oven setup features the reflective tantalum foil, so the changed crucible is the only modification. For approximately two weeks, the evaporation rate stayed rather stable and fluctuated around a value of $1.7 \mathrm{mg} \mathrm{h}^{-1}$. After the run, the oven showed no traces of lead oxide, while similar runs without the crucible modification and with the same oxygen pressure in the test stand clearly showed the formation of lead oxide. A photo of the oven tip after the experiment is shown on the lower right side in Fig. 3.

As the presented measurement is the first with this crucible design, no prior relation between deposition and evaporation rate

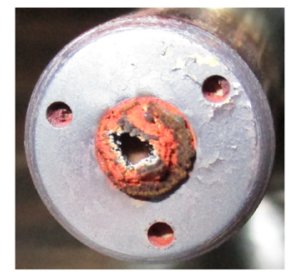

Normal setup (at GTS-LHC)

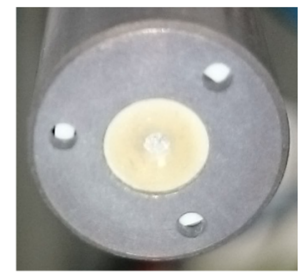

No heat refl. foil

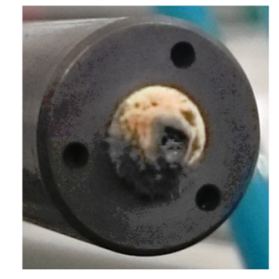

Normal setup (at OTS)

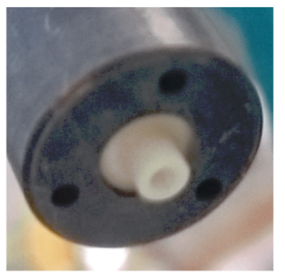

Crucible with beak
FIG. 3. Photos of the oven tip after a run at the GTS-LHC ion source in 2015 and after three different runs at the test stand, all within an oxygen pressure of $1 \times 10^{-5} \mathrm{mbar}$ and over the duration of two weeks. 


\section{Normal crucible}

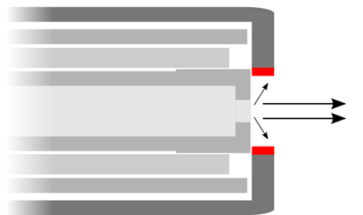

\section{Deposition on oven cover}

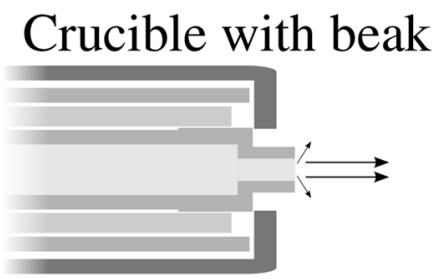

FIG. 4. Sketch of the oven with the normal crucible and the beak, showing the difference of the lead vapor exposure of the outer oven cover.

had been measured, which led to an overestimation of the evaporation rate during the measurement. While the achieved evaporation rate in this measurement was too low for actual operation at the GTS-LHC ion source, it is believed that a higher rate is achievable by choosing a higher heating power.

\section{CONCLUSION}

In this paper, data from the test stand are presented that hint that parts of the evaporation rate instabilities come from temperature instabilities of the oven itself and not from an inhomogeneous thermal profile. Possible causes of these instabilities were discussed. When the oven is operated without an internal heat reflective foil, its evaporation rate becomes more stable and displays no sudden drops. Additionally, the reduced insulation allows us to operate the oven within an oxygen pressure of $1 \times 10^{-5}$ mbar without any formation of lead oxide, while the oven in the usual setup would get clogged. This is a possible strategy to operate the oven for longer times at the GTS-LHC. However, the implications of running the oven with a higher power need to be studied, e.g., if the filament ages faster or if the increased thermal radiation could damage parts of the ion source.

Another way of avoiding the formation of a lead oxide blockage was demonstrated, that also works when the oven has its full insulation. Here, the crucible is shaped in a way that no lead vapor can be deposited on the outer oven cover. While for the setup without reflective foil evaporation rates that should be sufficient for operation at the GTS-LHC ion source have been observed, it remains to be tested if it is also possible with the modified crucible. Applying this technique at the source would be rather risk free except from the possibility that the beak could fall into the plasma chamber.

The results will help to modify the oven toward an increased stability that allows longer times of uninterrupted and reliable metal ion beam production and thereby could reduce the downtime during the LHC heavy ion program.

\section{REFERENCES}

${ }^{1}$ C. E. Hill, D. Küchler, R. Scrivens, D. Hitz, L. Guillemet, R. Leroy, and J. Y. Pacquet, AIP Conf. Proc. 749, 127-130 (2004).

${ }^{2}$ C. Fichera, F. Carra, D. Küchler, and V. Toivanen, Nucl. Instrum. Methods Phys. Res., Sect. A 901, 21-31 (2018).

${ }^{3}$ The official INFICON website, http://www.inficon.com; accessed 03 October 2017.

${ }^{4}$ T. Kövener, D. Küchler, and V. Toivanen, "Study of the lead evaporation from the oven of the GTS-LHC ion source," in Proceedings, 23rd International Workshop on ECR Ion Sources (ECRIS2018): Catania, Italy, September 10-14, 2018, Geneva, Switzerland, http://www.jacow.org, p. TUP21.

${ }^{5}$ R. Lang, J. Bossler, H. Schulte, and K. Tinschert, Rev. Sci. Instrum. 71, 651-653 (2000). 